\title{
Transforming OMFS through Artificial Intelligence and Machine Learning
}

\section{Neel Gupta*}

Post Graduate Resident, Department of Oral and Maxillofacial Surgery, Institute of Dental Studies and Technologies, Modinagar, Ghaziabad, Uttar Pradesh, India

*Corresponding Author: Neel Gupta, Post Graduate Resident, Department of Oral and Maxillofacial Surgery, Institute of Dental Studies and Technologies, Modinagar, Ghaziabad, Uttar Pradesh, India.
Received: September 24, 2021

Published: October 08, 2021

(C) All rights are reserved by Neel Gupta.

Keywords: Artificial Intelligence (AI); Machine Learning; Deep Learning; Neural Networks; Augmented Reality

The intelligence shown by computed machines is known as artificial intelligence (A.I.). It is also sometimes referred to as "machines that imitate cognitive functions associated with the human mind, including as learning and problem solving" [1]. AI is already present in our daily lives via smartphones and automobiles. It has now made its way into the field of surgery. Fortunately, as the area of robotic surgery has progressed, more doctors are becoming interested in the technology and the possibility for autonomous movements in procedures like interventional radiology, endoscopic surgeries, and Head and Neck surgeries. Many surgeons have been hesitant to accept robots because of the lack of motion controllers, or the feeling of touch; however, now that the actual potential of robotics has been realised, the surgical community's embrace of $\mathrm{AI}$ is more pertinent than ever.

How AI and Machine Learning helping surgeons?.

\section{Clinical decision-making assistance}

$\mathrm{AI}$ in healthcare can be beneficial in clinical decision support to assist surgeons make better and quicker judgments by recognising patterns in health problems that are registered significantly more precisely than the human brain. The time saved and the problems detected are critical in an industry where the time taken and decisions made can have life-changing consequences for patients.

Prediction of post operative complications using artificial neural networks

Artificial neural networks are made up of a large network of interconnected computer processors that analyse data using an automatic dynamic-state reaction to external inputs. Similar to the human brain, the ANN can identify patterns, handle data, and learn. Clinical data may be utilised to train neural network expert systems, which can then be employed in clinical scenarios to improve health-care system clinical tactics. Zhang., et al. used an artificial neural network to create a model that predicts postoperative face edema following third molar extraction with 98 percent accuracy [2]. Based on deep convolutional neural networks, In their study, Lee., et al. proposed that Deep Convolutional Neural Networksbased analysis of cephalometric radiograph pictures may be successfully utilised for differential diagnosis of orthognathic surgery indications with a 95.4-96.4 percent success rate [3].

\section{Radiodiagnosis through deep learning}

Furthermore, deep learning, a type of machine learning, is rapidly being used in the field of diagnosis and prediction in medical imaging, with promising outcomes. In the last two decades, major advancements in image identification utilising artificial intelligence systems have opened up a new route in the field of radiodiagnosis. AI has a particular edge in head and neck imaging modalities due to its unique capacity to learn. It can be used with other imaging modalities such as CBCT and MRI to detect minute deviations from normalcy that would otherwise go unnoticed by the human eye. Yang., et al. showed promising results for automated identification of jaw cysts and tumours in Orthopantomogram [4]. Kim., et al. suggested Machine learning methods showed superior performance compared to conventional statistical methods in anticipating BRONJ associated with dental extraction in patients taking bisphosphonates for osteoporosis [5]. 


\section{Conclusion}

In the existing market, AI has formed a trend, which will continue in the future. Despite the fact that AI benefits practitioners, a typical question that arises in their minds is, "Will AI replace practitioners in the future?" "It's not about humans being replaced by robots; it's about humans being enriched by machines". Robots and humans have different capabilities and weaknesses, and the symbiosis of the two will undoubtedly improve a surgeon's ability to handle challenging cases. AI represents the future of assisting doctors in integrating many disciplines of expertise in order to provide better clinical outcomes. Hereby author believes that oral and maxillofacial surgery residents should optimize themselves to comprehend its clinical utility, and start a discussion about how it may be used and controlled in everyday clinical practise.

\section{Bibliography}

1. Russell S and Norvig P. Artificial intelligence a modern approach third edition (Pearson, 2010).

2. Zhang W., et al. "Predicting postoperative facial swelling following impacted mandibular third molars extraction by using artificial neural networks evaluation". Scientific Report (2018): 12281.

3. Lee KS., et al. "Deep convolutional neural networks based analysis of cephalometric radiographs for diferential diagnosis of orthognathic surgery indications". Applied Science 10 (2020): 2124.

4. Yang H., et al. "Deep learning for automated detection of cyst and tumors of the jaw in panoramic radiographs". Journal of Clinical Medicine 9 (2020): 1839.

5. Kim DW., et al. "Machine learning to predict the occurrence of bisphosphonate-related osteonecrosis of the jaw associated with dental extraction: A preliminary report". Bone (2018): 207-214.

Volume 3 Issue 11 November 2021 (C) All rights are reserved by Neel Gupta. 